\title{
SURGICAL VS NONSURGICAL THERAPY ADJUNCTED WITH TETRACYCLINE LOCAL DRUG DELIVERY IN DIABETIC PATIENTS: A RANDOMIZED CLINICAL TRIAL
}

Aashutosh V. Karnik ${ }^{1}$, Mala Dixit Baburaj ${ }^{2}$

${ }^{1}$ Assistant Professor, Department of Periodontics, Bharati Vidyapeeth Deemed University Dental College and Hospital, Mumbai, India

${ }^{2}$ Professor and Head, Department of Periodontics, Nair Hospital Dental College, Mumbai, India

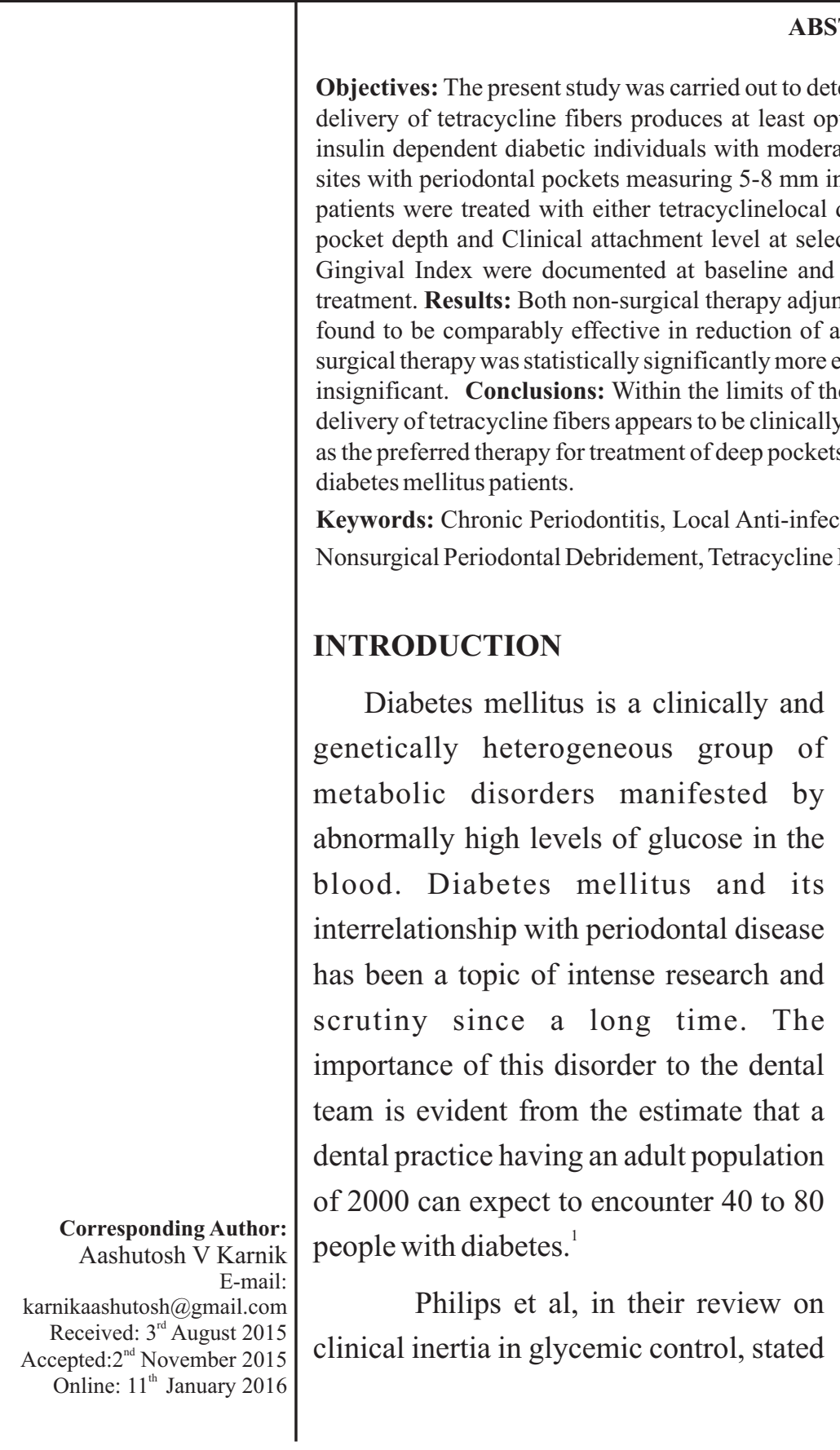

\section{ABSTRACT}

Objectives: The present study was carried out to determine if non-surgical therapy adjuncted with local drug at least optimally comparable results as surgical therapy in nonth moderate to severe periodontitis. Method and Materials: 80 40 controlled non-insulin dependent diabetes mellitus parameters except Probing Pocket Depth, for which surgical therapy was statistically significantly more efficacious $(p<0.01)$, though clinically the difference was insignificant. Conclusions: Within the limits of the study, non-surgical therapy adjuncted with local drug delivery of tetracycline fibers appears to be clinically as efficacious as surgical therapy and may be employed 列 Keywords: Chronic Periodontitis, Local Anti-infective Agents, Non-insulin Dependent Diabetes Mellitus, Nonsurgical Periodontal Debridement, Tetracycline Hydrochloride

\section{INTRODUCTION} enetically heterogeneous group of metabolic disorders manifested by 政 blood. Diabetes mellitus and its scrutiny since a long time. The team is evident from the estimate that a dental practice having an adult population of 2000 can expect to encounter 40 to 80 people with diabetes.

Philips et al, in their review on that data from the United States suggest that of the $65 \%$ of the patients diagnosed with diabetes, only $73 \%$ are prescribed pharmacologic therapy and only $33 \%$ of those thus treated achieve a HbA1C (Glycated Hemoglobin A1C) value of less than $7 \%$ by the American Diabetes Association (ADA) goal. ${ }^{2}$ Rees, in a monograph, stated that the long term glycemic control of even the so called 'controlled' diabetics may not be very consistent by rigid standards, yet the patient's physician will provide medical clearance to perform periodontal therapy because the patient is likely to tolerate the procedure without undue difficulty. In this circumstance the Periodontist should 
proceed with caution since inadequate diabetes mellitus control can adversely affect the severity of the periodontal disease response; the patient's wound healing capacity and the ability of the patient to withstand both emotional and physical stress. All these factors can result in a compromised clinical outcome. The clinician should insist that the patient achieve and sustain a highly effective level of oral physiotherapy, and in most instances a nonsurgical approach to periodontal therapy is preferred in individuals with diabetes mellitus, with or without the use of appropriate antibiotic therapy. ${ }^{3}$

Heitz-Mayfield, in a meta-analysis of three systematic review papers that reviewed all combined randomized controlled trials comparing scaling and root planing with open flap debridement, demonstrated that non-surgical therapy produces changes comparable to surgical therapy in clinical attachment level. ${ }^{4}$

Tetracycline fiber therapy has been shown to produce greater pocket depth reduction, attachment level gain and improvement in clinical response than and in addition to the effects that occur due to prophylaxis and improved home care. ${ }^{5}$ Pavia, Nobile and Angelillo in a meta-analysis demonstrated a greater change in terms of probing depth and attachment level when data was pooled from studies in which tetracycline was administered as an adjunct to scaling and root planing. The meta-analysis concluded that results of various studies have documented that local delivery of tetracycline improves the clinical outcome of traditional treatment of scaling and root planing and should be considered particularly as an adjunct to it. ${ }^{6}$

The present study was carried out to determine if non-surgical therapy adjuncted with local drug delivery of tetracycline fibers produces at least optimally comparable results as surgical therapy in controlled non-insulin dependent diabetic individuals with moderate to severe periodontitis. These observations may point out if non-surgical therapy adjuncted with tetracycline local drug delivery may be used as the preferred form of therapy in lieu of flap surgery in treatment of deep pockets in diabetic individuals, considering the fact that their wound healing patterns and overall body biochemistry segregate them from other patients with periodontal disease.

\section{METHOD AND MATERIALS}

\section{Study Design}

The study was a parallel randomized trial carried out at Nair Hospital Dental College in Mumbai from $1^{\text {st }}$ January, 2008 to $31^{\text {st }}$ August, 2009. It was independently reviewed and approved by the human subjects ethics board of the institute and was conducted in accordance with the World Medical Association Declaration of Helsinki 1975, as revised in 2000. A total of 40 patients were selected for this study from the outpatient department and their informed written consent was taken prior to the study. Patients were selected using the following selection criteria: 1) Patients with controlled non-insulin dependent diabetes mellitus (random blood glucose 120-140 mg/dl); 2) Patients who had not taken antibiotics for the past 6 weeks; 3 ) Patients who had moderate to severe periodontitis with pockets between $5 \mathrm{~mm}-8 \mathrm{~mm}$ at minimum 2 sites measured after a minimum of 15 days after scaling and root planing. To minimize the surgical area, sites close to each other were preferred. The following patients were excluded from selection: 1) Patients who had significant systemic history other than diabetes mellitus; 2) Patients who gave history of allergy to tetracycline; 3) Pregnant patients, lactating mothers or females taking oral contraceptives; 4) Smokers; 5) Patients who were showing signs of oral candidiasis.

Patients were subjected to detailed case history, examination and counselling for maintenance of good oral hygiene, prior to nonsurgical periodontal 
debridement. Modified Bass method was taught for toothbrushing and appropriate interdental cleansing aids were prescribed. The type of diabetes was established by using the following criteria: 1) Age of onset $>25$ years; obesity; 2) Treatment primarily based on diet control, exercise and oral hypoglycemic agents with only occasional emergency use of supplementary insulinand; 3) Physician's consent. ${ }^{1}$ Their glycemic control was established by using the following criteria: 1) Physician's consent; 2) Random blood glucose testing (120-140 mg/dl was considered acceptable). ${ }^{8}$

After enrollment, the investigatorsrandomly allocated the subjects into two groups: Group 'NS + LDD' (Non-Surgical therapy + Local Drug Delivery) and Group 'S' (Surgical therapy). Simple randomization was carried out by the investigators using the chit method, after writing NS+LDD and S on 20 chits each, folding the chits, putting them in a box and mixing them well. Two sites fulfilling the above mentioned criteria were identified in each patient and were named 'a' and ' $b$ ', to be monitored at the aforementioned time intervals. The deepest sites around the selected teeth were included in the study. The baseline readings of Probing Pocket Depth (PPD) and Clinical Attachment Level (CAL) were recorded with a UNC-15 Probe after a minimum of 15 days subsequent to scaling and root planing and were designated as 'al' and 'bl'. The subsequent readings of PPD and CAL were designated as follows: 1) 'a2', 'b2' at 4 weeks; 2) 'a3', 'b3' at 6 weeks; 3) 'a4', 'b4' at 9 weeks; 4) 'a5', 'b5' at 12 weeks. Along with PPD and CAL, Plaque Index (PI) ${ }^{9}$ and Gingival Index (GI) ${ }^{10}$ were also measured post treatment at the same time intervals.

\section{Intervention Groups}

\section{GROUP' NS + LDD'}

In Group 'NS + LDD', tetracycline resorbable fiber local drug delivery was done using Periodontal Plus $\mathrm{AB}^{\circledR}$ fibers. Periodontal Plus $\mathrm{AB}^{\circledR}$ fibers are made up of
$25 \mathrm{mg}$ of pure fibrillar collagen containing approximately $2 \mathrm{mg}(1.7 \pm 0.25 \mathrm{mg})$ of evenly impregnated tetracycline hydrochloride that functions as a local anti-infective agent providing continuous release of tetracycline for a minimum of 10 days. These fibers release tetracycline at a rate of approximately 2 $\mu \mathrm{g} / \mathrm{mg}-\mathrm{hr}$ in the periodontal pocket with average concentration of $1500 \mu \mathrm{g} / \mathrm{ml}$ per site during the first 10 days' treatment period.

The sites receiving fiber therapy were carefully isolated using cotton rolls. Tetracycline impregnated collagen fibers were taken in a dappen dish and moistened with sterile normal saline (Figure 1a) and gently packed in the periodontal pockets. The fibers were inserted in the deepest sites and successive layers were placed till all subgingival areas were completely

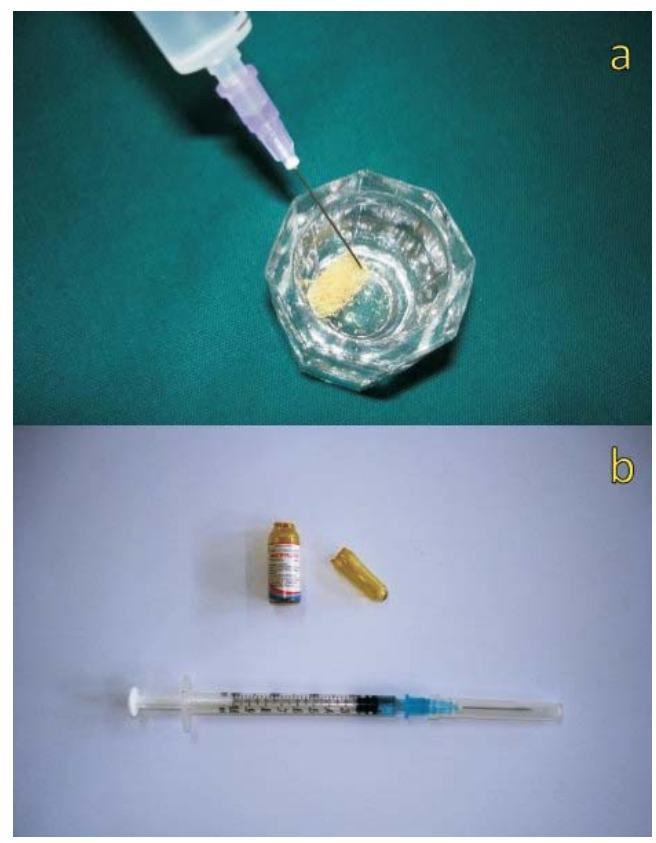

Figure 1: (a) Golden-yellow tetracycline resorbable fibers being moistened with sterile saline in a sterile dappen dish;

(b) Blue colored cyanoacrylate tissue adhesive withdrawn from sterile ampoule into a syringe

filled to the gingiva margin. The back of a Universal Curette $(\mathrm{U} 4 \mathrm{R} / 4 \mathrm{~L})$ was used to insert fibers in the periodontal pockets. 
To retain the fibers in the pocket a thin layer of tissue adhesive, Amcrylate, was used to seal the gingival sulcus. Amcrylate is sterile tissue adhesive (Iso amyl 2-cyanoacrylate) in the form of a monomer that polymerizes on contact with moisture and solidifies within less than 5-10 seconds. The Amcrylate glass ampoule was cut and the Amcrylate solution was drawn into the syringe provided along with the Amcrylate uni-pack (Figure 1b). Amcrylate was applied in a thin layer and the excess was removed with dry swab immediately.

Patients were given following instructions till the time cyanoacrylate adhesive was not removed:1) Do not brush or floss the treated area; 2) Do not eat hard or crunchy foods; 3 ) Do not chew gum or sticky foods; 4)Avoid touching the treated area with tongue or fingers; 4) Report back if the adhesive dressing falls out before 7 days; 5) Although some mild to moderate sensitivity is normal after treatment with Periodontal Plus AB fibers, report back promptly if pain, swelling or other problems occur.

Approximately 4 days following the placement of tetracycline fibers, the patients were checked for fiber retention and any adverse soft tissue reaction. If the fibers were dislodged or lost, they were replaced. Cyanoacrylate adhesive was removed after 10 days of application. There was no need to remove the fibers as they were bio-resorbable.

\section{GROUP' $S^{\prime}$}

Patients in this group were subjected to Modified Flap Operation (Kirkland Flap) in selected sites. Anesthesia was achieved by infiltrations and/or nerve blocks using Inj. $2 \%$ Lignocaine Hydrochloride with 1 : 100, 000 Epinephrine. Kirkalnd sulcular incisions were made andfull thickness mucoperiosteal flaps were reflected taking care to minimize the extent of surgical area.
Thorough debridement was carried out to remove granulation tissue and any residual subgingival calculus. Root planing was carried out using curettes. The pocket lining was trimmed from within using scissors. The area was flushed with saline and sutured using $4-0$ black braided silk. Periodontal dressing was placed using Coe-Pak.

The patients were given routine post-surgical instructions. They were prescribed Tab. Diclofenac potassium $50 \mathrm{mg}$ B.D. for 3 days and Mouthwash Chlorhexidine $0.20 \%$ B.D. for 7 days. The dressing was removed after 7 days and the patients were asked to gradually resume function and oral hygiene practices in the operated sites.

\section{STATISTICALANALYSIS}

The null hypothesis was that the NS + LDD and the $S$ Group had statistically significant differences in mean values of PPD, CAL, PI and GI. After clinical observations, the data was collected and subjected to statistical analysis. Unpaired $t$ Test was used to compare values within the groups. One way ANOVA was used for comparing the changes in PPD and CALbetween the two groups.For comparing the changes in PI and GI, Mann-Whitney U test was used. IBM SPSS 22 was used for the statistical analysis.

\section{RESULTS}

Of the 40 originally selected patients ( 24 males and 16 females), 3 dropped out of the NS + LDD Group while one was not considered for the study as the oral hygiene levels were unacceptable. 4 patients dropped out of the S Group, thus bringing the total to 32 patients, 16 in Group NS + LDD and 16 in Group S. The total number of sites monitored was 64 (Baseline data in Table 1). Figure 2 provides CONSORT Flow Diagram for the study.

Mean Probing Pocket Depth (PPD) scores for Group NS + LDD at baseline, 4 weeks, 6 weeks, 9 weeks and 12 weeks post treatment were $5.75 \pm 0.61$, 
Table 1: Baseline Data of Group NS+LDD and Group S

\begin{tabular}{|c|c|c|c|c|c|c|c|c|c|c|c|c|}
\hline \multirow[t]{3}{*}{ Number } & \multicolumn{6}{|c|}{ Group NS+LDD } & \multicolumn{6}{|c|}{ Group S } \\
\hline & \multicolumn{2}{|c|}{$\begin{array}{c}\text { PPD in } \\
\text { millimeters }\end{array}$} & \multicolumn{2}{|c|}{\begin{tabular}{|c|} 
CAL in \\
millimeters
\end{tabular}} & \multirow[t]{2}{*}{ PI } & \multirow[t]{2}{*}{ GI } & \multicolumn{2}{|c|}{$\begin{array}{c}\text { PPD in } \\
\text { millimeters }\end{array}$} & \multicolumn{2}{|c|}{$\begin{array}{c}\text { CAL in } \\
\text { millimeters }\end{array}$} & \multirow[t]{2}{*}{ PI } & \multirow[t]{2}{*}{ GI } \\
\hline & Site ' $a$ ' & Site 'b' & Site 'a' & Site 'b' & & & Site 'a' & Site 'b' & Site 'a' & Site ' $b$ ' & & \\
\hline 1 & 5 & 5 & 6 & 6 & 1.23 & 1.21 & 6 & 5 & 6 & 5 & 0.81 & 1.12 \\
\hline 2 & 6 & 5 & 6 & 5 & 1.13 & 1.25 & 5 & 6 & 5 & 7 & 1.13 & 1.52 \\
\hline 3 & 5 & 6 & 6 & 7 & 0.93 & 1.34 & 5 & 5 & 5 & 5 & 1.49 & 1.48 \\
\hline 4 & 5 & 6 & 6 & 7 & 0.95 & 1.32 & 6 & 6 & 6 & 6 & 1.15 & 1.21 \\
\hline 5 & 6 & 5 & 6 & 5 & 1.23 & 1.24 & 5 & 6 & 5 & 6 & 0.84 & 1.25 \\
\hline 6 & 5 & 6 & 6 & 5 & 0.73 & 1.37 & 7 & 8 & 7 & 8 & 0.65 & 1.17 \\
\hline 7 & 7 & 6 & 7 & 6 & 0.81 & 1.93 & 5 & 5 & 6 & 5 & 0.89 & 1.11 \\
\hline 8 & 5 & 7 & 6 & 8 & 1.42 & 1.58 & 7 & 7 & 7 & 7 & 1.28 & 1.27 \\
\hline 9 & 6 & 6 & 6 & 6 & 0.86 & 1.35 & 5 & 6 & 5 & 6 & 1.19 & 1.43 \\
\hline 10 & 6 & 5 & 7 & 6 & 1.74 & 1.67 & 6 & 6 & 6 & 6 & 1.43 & 1.53 \\
\hline 11 & 6 & 5 & 6 & 5 & 1.36 & 1.28 & 7 & 8 & 7 & 8 & 0.64 & 1.25 \\
\hline 12 & 5 & 7 & 5 & 7 & 0.99 & 1.38 & 6 & 6 & 6 & 6 & 1.32 & 1.13 \\
\hline 13 & 6 & 5 & 6 & 5 & 0.72 & 1.21 & 5 & 5 & 5 & 5 & 0.64 & 1.26 \\
\hline 14 & 7 & 8 & 7 & 8 & 1.27 & 1.33 & 6 & 5 & 6 & 5 & 0.89 & 1.25 \\
\hline 15 & 6 & 6 & 6 & 6 & 0.91 & 1.24 & 5 & 6 & 5 & 6 & 1.45 & 1.21 \\
\hline 16 & 5 & 5 & 7 & 5 & 0.83 & 1.27 & 5 & 5 & 5 & 5 & 0.93 & 1.04 \\
\hline
\end{tabular}

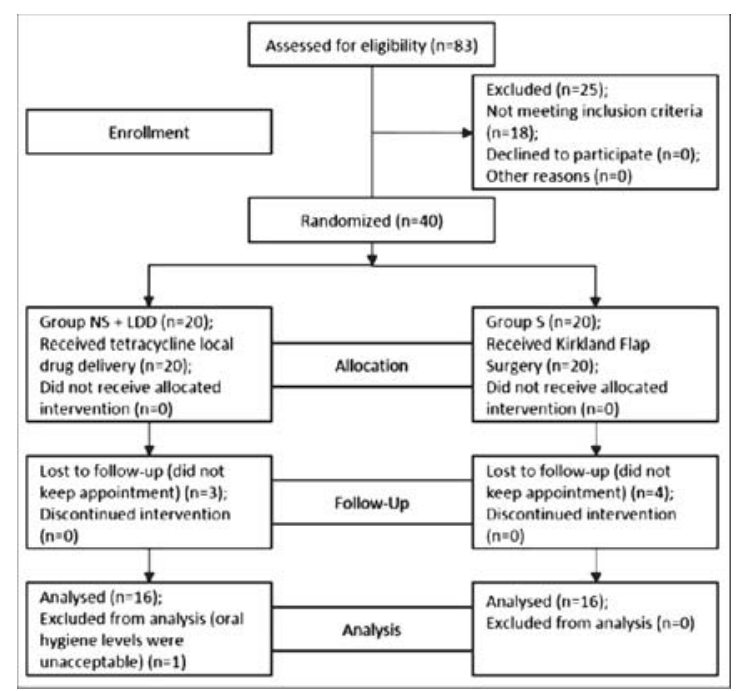

Figure 2: CONSORT Flow Diagram of the progress through enrolment, intervention allocation, follow-up and analysis of a parallel-randomized trial of two groups

$2.66 \pm 0.30,2.66 \pm 0.30,2.66 \pm 0.30$ and $2.66 \pm 0.30$ respectively. In Group S, the mean PPD measurement at baseline, 4 weeks, 6 weeks, 9 weeks and 12 weeks post treatment were $5.84 \pm 0.83,1.91 \pm 0.46,1.91 \pm$ $0.46,1.91 \pm 0.46$ and $1.91 \pm 0.46$ respectively. Figure 3 describes the changes in mean PPD over the course of the study at both sites in both groups. In both groups at

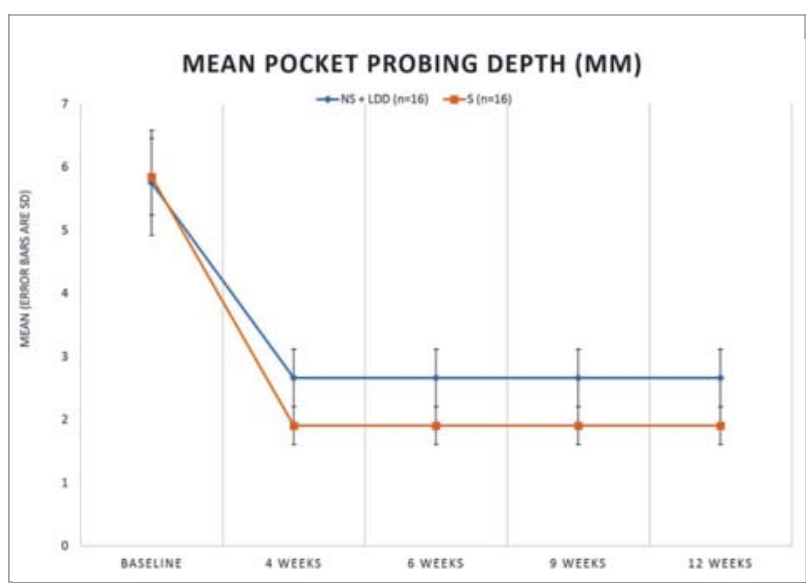

Figure 3: Mean PPD across Group NS+LDD and Group S

both sites, statistically significant difference was found in PPD values between baseline and 4 weeks $(\mathrm{p}<0.01)$. No statistically significant difference was found between 4 to 6 weeks, 6 to 9 weeks and 9 to 12 weeks in both sites $(p>0.05)$. On comparing changes inmean scores of PPD between the two groups using one way ANOVA, except for the baseline, there was a statistically significant difference between Group NS + LDD and Group S (F ratio significantly more than 1) (Figure 4). 


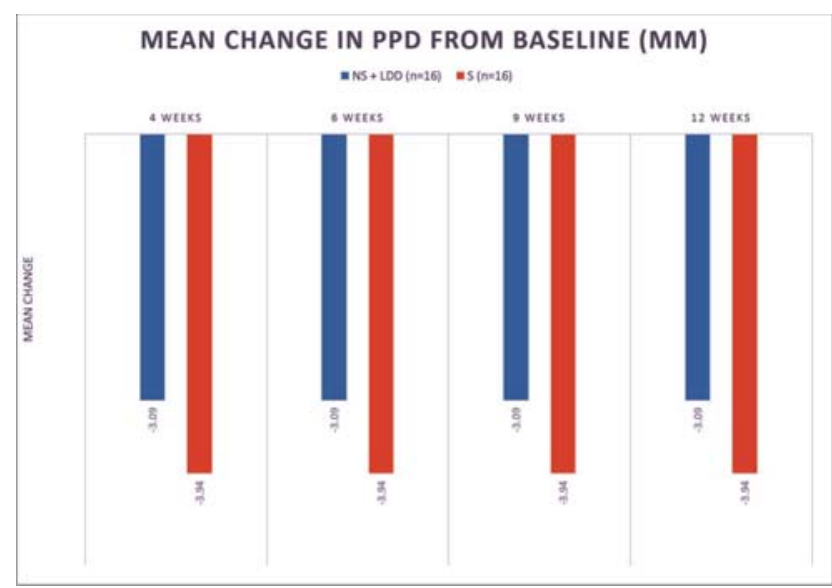

Figure 4: Mean PPD Change across Group NS+LDD and Group S

Mean Clinical Attachment Level (CAL) scores for Group NS + LDD at baseline, 4 weeks, 6 weeks, 9 weeks and 12 weeks post treatment were $6.16 \pm 0.57$, $3.06 \pm 0.36,3.06 \pm 0.36,3.06 \pm 0.36$ and $3.09 \pm 0.38$ respectively. In Group S, the mean CAL measurements at baseline, 4 weeks, 6 weeks, 9 weeks and 12 weeks post treatment were $5.88 \pm 0.81,3.06 \pm 0.36,3.06 \pm$ $0.31,3.06 \pm 0.31$ and $3.09 \pm 0.38$ respectively. Figure 5 describes the changes in mean CAL over the course of

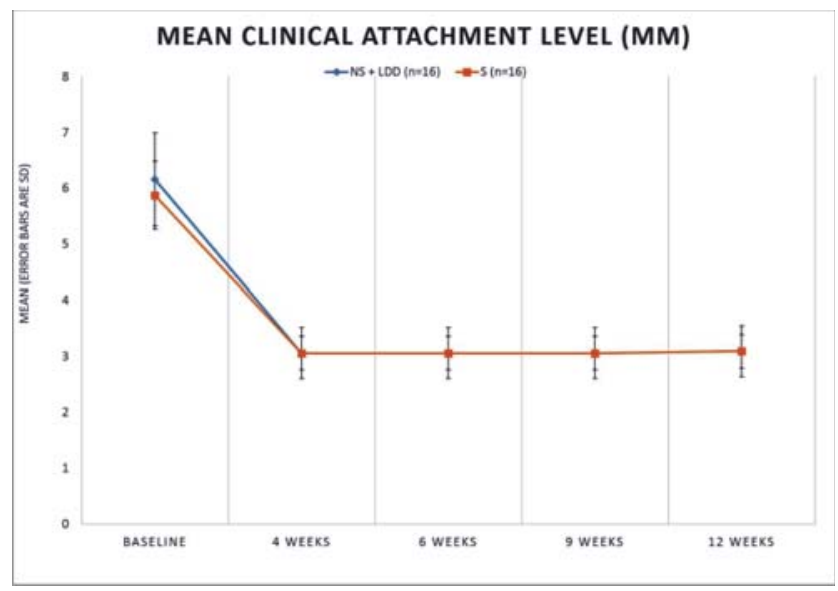

Figure 5: Mean CAL across Group NS+LDD and Group S

the study at both the sites in both the groups. In both the groups at both the sites, statistically significant difference was found in CAL values between baseline and 4 weeks $(p<0.01)$. No statistically significant difference was found between 4 to 6 weeks, 6 to 9 weeks and 9 to 12 weeks in both sites $(p>0.05)$. On comparing mean scores of CAL between the two groups using one way ANOVA, there was no statistically significant difference between Group NS + LDD and Group $\mathrm{S}$ (F ratio close to 1 ), at any time interval (Figure 6).

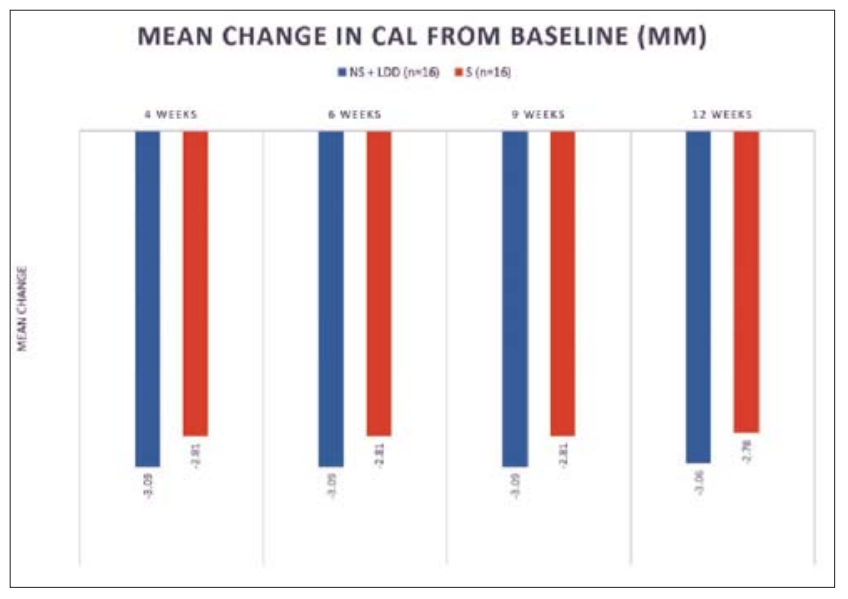

Figure 6: Mean CAL Change across Group NS+LDD and Group S

The mean Plaque Index (PI) score for Group $\mathrm{NS}+\mathrm{LDD}$ was $1.07 \pm 0.29$ at baseline, $0.86 \pm 0.21$ at 4 weeks, $0.74 \pm 0.21$ at 6 weeks, $0.64 \pm 0.22$ at 9 weeks and $0.56 \pm 0.21$ at 12 weeks post treatment. The mean PI score for Group S was $1.05 \pm 0.30$ at baseline, $0.96 \pm$ 0.29 at 4 weeks, $0.87 \pm 0.30$ at 6 weeks, $0.77 \pm 0.29$ at 9 weeks and $0.66 \pm 0.29$ at 12 weeks post treatment. The mean difference in PI at baseline and 4, 69 and 12 weeks was statistically highly significant in both the groups $(\mathrm{p}<0.01)$. On comparing mean scores of PI between the two groups using the Mann-Whitney U Test, there was no statistically significant difference in the mean scores at baseline and at the follow-up intervals $(\mathrm{p}>0.05)$ (Figure 7). The mean Gingival Index (GI) score for Group NS $+L D D$ was $1.37 \pm 0.20$ at baseline, $0.78 \pm 0.15$ at 4 weeks, $0.57 \pm 0.13$ at 6 weeks, $0.49 \pm 0.19$ at 9 weeks and $0.37 \pm 0.17$ at 12 weeks post treatment. The mean GI score for Group S was $1.26 \pm 0.15$ at baseline, $0.74 \pm 0.16$ at 4 weeks, 0.55 


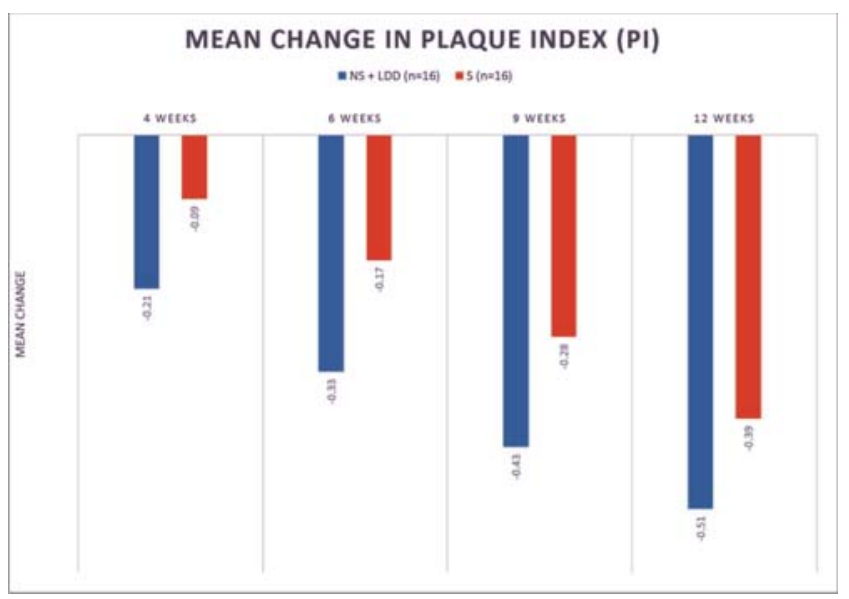

Figure 7: Mean PI Changes across Group NS+LDD and Group S

\pm 0.18 at 6 weeks, $0.44 \pm 0.16$ at 9 weeks and $0.33 \pm 0.16$ at 12 weeks post treatment. The mean difference in GI at baseline and 4, 69 and 12 weeks post treatment was statistically highly significant $(\mathrm{p}<0.01)$ in both the groups. On comparing mean scores of GI between the two groups using the Mann-Whitney U Test, there was no statistically significant difference in the mean scores at baseline and at the follow-up intervals $(\mathrm{p}>0.05)$ (Figure 8).

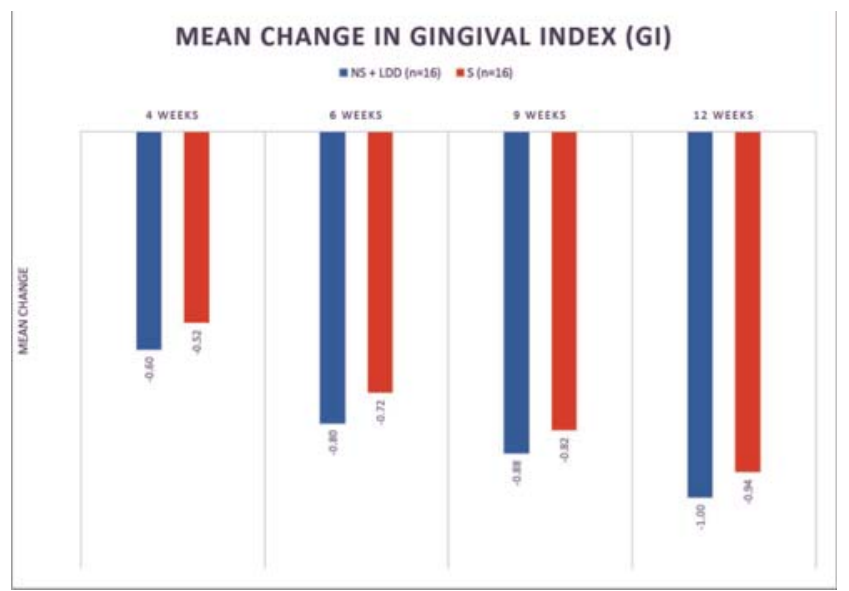

Figure 8: Mean GI Changes across Group NS+LDD and Group S

\section{DISCUSSION}

In both the groups, statistically significant reduction in Probing Pocket Depth (PPD) was observed from the baseline to 4, 6, 9 and 12 weeks post treatment. There was a statistically significant difference observed when the two groups were compared to each other. This demonstrates that whereas, both the treatment modalities are effective in reducing in probing pocket depths, the surgical therapy seems to be more effective in doing so than the non-surgical therapy, even when adjuncted with tetracycline local drug delivery. This phenomenon can be explained on the basis of the fact that surgical therapy resulted in more gingival recession than non-surgical therapy adjuncted with local drug delivery. Even so, the 12 weeks mean values of non-surgical therapy adjuncted with tetracycline local drug delivery fall within the limits of periodontal health and hence can be deemed acceptable. The patients treated by non-surgical therapy adjuncted with tetracycline local drug delivery succeeded in maintaining acceptably good oral hygiene with the help of appropriate cleansing aids due to improved periodontal parameters, as demonstrated by the comparable plaque indices of the two groups at the end of the study period.

In both groups, statistically significant reduction in Clinical Attachment Level (CAL) was observed from the baseline to 4, 6, 9 and 12 weeks post treatment. There was a statistically insignificant difference observed when the two groups were compared to each other. This demonstrates that both the treatment modalities are equally effective in gaining clinical attachment level. This once again reinforces the fact that surgical therapy results in more gingival recession than non-surgical therapy adjuncted with local drug delivery.

Also, the reductions obtained in PPD and CAL at the end of 4 weeks were maintained in both the groups till the end of the study period, as demonstrated by insignificant differences at 4, 6, 9 and 12 weeks post treatment within each groups.

The results of the present study in connection with PPD and CAL are in agreement with long-term (12 
months to 5 years) trials. ${ }^{11-17}$ The present study, however, involved special population i.e. diabetic patients. Also, all the above mentioned trials did not employ any local drug delivery that served as an adjunct to non-surgical therapy. Nevertheless, an overall agreement with the results of these long-term trials could be demonstrated.

In both groups, statistically significant reduction in Plaque Index (PI) and Gingival Index (GI) was observed from the baseline to 4, 6, 9 and 12 weeks post treatment. However there was no statistically significant difference observed when the two groups were compared to each other. This demonstrates that both the treatment modalities result in comparable reduction in the plaque scores of the patients. A general trend of progressive decline in plaque index scores over the duration of the study was seen. This could be due to the repeated reinforcement of oral hygiene habits in recall visits and overall general improvement in periodontal parameters.

The reduction in PI is in agreement with many longitudinal periodontal therapy trials that compare non-surgical therapy with surgical therapy. ${ }^{11-13,15,18-23}$ The reduction in GI is also in agreement with many longitudinal periodontal therapy trials that compare non-surgical therapy with surgical therapy. ${ }^{11,12,14,15,20-22,24}$

One of the primary limitation of the present trial was the relatively short duration of study. A trial with a long term follow up can further substantiate the findings of this study. Also, a trial that takes into account other local contributing etiologies like furcations can help in eliminating other confounding factors.

In summary, both non-surgical therapy adjuncted with local drug delivery and surgical therapy were found to be effective in reducing PPD, CAL, PI and GI. No statistically significant difference was found in the efficacy of the two therapies for any of the parameters investigated except PPD, for which surgical therapy was found to be significantly more efficacious of the two. However, the reduction in probing pocket depth with non-surgical therapy adjuncted with local drug delivery was found to be clinically sufficient to restore the health of the periodontal tissues.

\section{CONCLUSION}

Based on these observations, within the limits of the study, the following conclusions can be drawn:

- Non-surgical therapy adjuncted with local drug delivery of tetracycline resorbable fibers appears to be clinically as efficacious as surgical therapy for treatment of deep pockets measuring $5-8 \mathrm{~mm}$ in controlled non-insulin dependent diabetes mellitus patients.

- Non-surgical therapy adjuncted with local drug delivery may be employed as the preferred therapy in deep pockets measuring $5 \mathrm{~mm}-8 \mathrm{~mm}$ in individuals with controlled type II diabetes mellitus.

\section{REFERENCES}

1. Rose L, Steinberg B, Atlas S. Periodontal Management of the Medically Compromised Patient. Periodontology 2000 1995;9:165-175

2. Phillips L, Branch W, Cook C, Doyle J, El-Kebbi I, Gallina D. Clinical Inertia. Ann Intern Med 2001;135:825-834

3. Rees T. Periodontal management of patients with diabetes mellitus. Periodontology 2000;23:63-72

4. Heitz-Mayfield LJA. How effective is surgical therapy compared with nonsurgical debridement? Periodontology 2005;37:72-87

5. Research, Science and Therapy Committee of American Academy of Periodontology. The role of controlled drug delivery in periodontitis. J Periodontol 2000;71:125-140

6. Pavia M, Nobile C, Angelillo I. Meta-Analysis of Local Tetracycline in Treating Chronic Periodontitis. J Perodontol 2003;74(6):916-932

7. Mealey B, Oates T. Diabetes Mellitus and Periodontal Diseases: AAP- Commissioned Review. J Periodontol 2006; 77:1289-1303 
8. Mealy B, Ocampo G. Diabetes mellitus and periodontal disease. Periodontology 2000 2007;44:127-153

9. Turesky S, Gilmore N, Glickman I. Reduced plaque formation by chlormethyl analogue of Vitamin C. J Periodontol 1970;41: 41-43

10. Loe $H$. The gingival index, the plaque index and the retention index systems. J Periodontol 1967;38(suppl):610

11. Lindhe J, Westfelt E, Nyman S, Socransky S, Heijl L, Bratthall G. Healing following surgical / non-surgical treatment of periodontal disease. A clinical study. J Clin Periodontol 1982;9:115-128

12. Pihlstrom B, McHugh R, Oliphant T, Ortiz-Campos C. Comparison of surgical and non-surgical treatment of periodontal disease. A review of current studies and additional results after 61/2 years. J Clin Periodontol 1983;10:524-541

13. Lindhe J, Westfelt E, Nyman S, Socransky S, Haffajee A. Long-term effect of surgical / non-surgical treatment of periodontal disease. J Clin Periodontol 1984;11:448-458

14. Lindhe J, Nyman S. Scaling and granulation tissue removal in periodontal therapy. J Clin Periodontol 1985;12:374-388

15. Isidor F, Karring $\mathrm{T}$. Long-term effect of surgical and nonsurgical periodontal treatment. A 5-year clinical study. J Periodontal Res 1986;21:462-472

16. Ramfjord S, Caffesse R, Morrison E, Hill R, Kerry G, Appleberry E. Four modalities of periodontal treatment compared over 5 years. J Clin Periodontol 1987;14:445-452
17. Kaldahl W, Kalkwarf K, Patil K, Molvar M, Dyer J. Long-term evaluation of periodontal therapy: Response to 4 therapeutic modalities. J Periodontol 1996;67:93-102

18. Zamet J. A comparative clinical study of three periodontol surgical techniques. J Clin Periodontol 1975;2:87-97

19. Waite I. A comparison between conventional gingivectomy and a nonsurgical regime in the treatment of periodontitis. J Clin Periodontol 1976;3:173-185

20. Pihlstrom B, Oritz-Campos C, McHugh R. Randomized fouryear study of periodontal therapy. J Periodontol 1981;52:227242

21. Westfelt E, Bragd L, Socransky S, Haffajee A, Nyman S, Lindhe J. Improved periodontal conditions following therapy. J Clin Periodontol 1985;12:283-293

22. Becker W, Becker B, Ochsenbein C. A longitudinal study comparing scaling, osseous surgery and modified Widman procedures. Results after one year. J Periodontol 1988;59:336351

23. Kaldahl W, Kalkwarf K, Patil K, Molvar M. Evaluation of four modalities of periodontal therapy. Gingival suppuration and supragingival plaque. J Clin Periodontol 1990;17:642-649

24. Kalkwarf K, Kaldahl W, Patil K, Molvar M. Evaluation of gingival bleeding following four types of periodontal therapy. J Clin Periodontol 1989;16:601-608 